Reprod. Nutr. Dévelop., 1981, 21 (2), 177-183.

\title{
Adaptation des TRNA ef optimisation de la traduction
}

\author{
par J. P. GAREL, G. CHAVANCY, A. CHEVALLIER, A. FOURNIER, G. MARBAIX*, \\ G. HUEZ *
}

\author{
Laboratoire associé au C.N.R.S. no 92, \\ Déportement de Biologie générale ef oppliquée, \\ Université Cl. Bernard-Lyon-1, 69622 Villeurbanne Cedex France \\ * Dépariement de Biologie moléculaire \\ Université Libre de Bruxelles, Rhode St-Genese, Belgique.
}

Summary. IRNA adaptation in optimizing translation.

The intracellular level of each tRNA species is adjusted to the codon frequency of the mRNA being decoded. This was first observed in such highly differentiated cells as the silk gland of Bombyx mori, which produces fibroin and sericin, and the rabbit reticulocyte. IRNA adaptation also occurs in other cell types from $E$. coli to mammalian cells. Regardless of the mechanism regulating IRNA biosynthesis, we believe that IRNA adaptation is the basic step optimizing chain elongation at the ribosomal level. We propose the system of trial and error as a working model for the ribosome. This model clarifies the correlations between isoaccepting tRNA levels and codon frequencies, as well as the effect of tRNA pool balance on mean elongation rate and non-uniform individual elongation rate (depending on whether codons are rare or abundant) for fibroin mRNA translated in a reticulocyte cell-free system.

\section{Introduction.}

La distribution intracellulaire des IRNA n'est pas quelconque, mais adaptée aux synthèses protéiques en cours. Dans des systèmes cellulaires spécialisés (cristallin de Veau, glande séricigène de Ver à soie), nous avons montré qu'il existe une corrélation étroite entre la composition de la population des IRNA ef la composition en acides aminés des protéines majeures, cristallines, fibroïne et séricine respectivement (Garel, Virmaux et Mandel, 1970 ; Chavancy, Daillie et Garel, 1971). Ce type de relation appelée adaptation des $t R N A$ est généralisable à l'ensemble des organismes uni- et pluricellulaires (Garel, 1974).

En fait, l'existence de cette corrélation repose sur un ajustement entre les concentrations de chaque espèce de tRNA - isoaccepteurs compris ou iso-łRNA - et la fréquence des codons synonymes du (ou des) mRNA en voie de traduction. La partie postérieure (ou sécréteur) de la glande séricigène de Bombyx mori a grandement facilité la mise en évidence de ce fait (Daillie, 1977 ; Prudhomme et Couble, 1979). Au cours du $5^{\mathrm{e}}$ et dernier âge larvaire, la glande séricigène entre dans une phase de sécrétion intense de fibroïne caractérisée par une composition déséquilibrée en acides aminés 
(46 p. 100 de glycine, 29 p. 100 d'alanine, 12 p. 100 de sérine) codés par un choix particulier de codons : GGU et GGA pour la glycine, GCU pour l'alanine et UCA pour la sérine (Suzuki et Brown, 1972). Une analyse exhaustive tant quantitative que structurale des tRNA majeurs du sécréteur, en particulier grâce à la cartographie électrophorétique, permit de conclure à une corrélation entre la population des tRNA ${ }_{1,2}^{\text {Gly }}$, tRNA $A_{2}^{\text {Ala }}$ et $t R N A_{2}^{\text {Ser }}$ et la fréquence des codons du messager de la fibroïne pendant la phase de croissance (Garel, 1976 ; Garel, Garber et Siddiqui, 1977).

\section{Résultats et discussion.}

1. Généralisation de l'adaptation des IRNA à la fréquence des codons.

L'existence d'une corrélation étroite entre la distribution des iso-tRNA dans le sécréteur du Ver à soie et la fréquence des codons synonymes du mRNA de la fibroïne peut être étendue d̀ d'autres systèmes spécialisés, à condition de disposer d'informations aussi précises que possible sur la distribution de tRNA à anticodon connu et sur celle des codons du messager prépondérant ou d'un échantillon du «messager moyen $\gg$.

C'est le cas du colibacille. La figure 1 montre une corrélation significative entre la distribution des tRNA et celle des codons de divers mRNA connus aujourd'hui chez $E$. coli $(r=0,82)$. La distribution des iso-tRNAGlu et iso-tRNA ${ }^{\text {Lys }}$ est complètement inversée par rapport à celle des animaux supérieurs, mais toujours corrélée à celle des codons GAA et AAA très abondants chez cette bactérie.

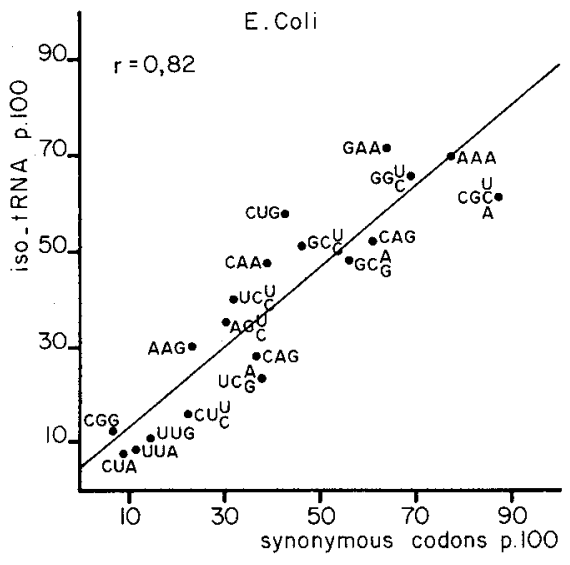

FIG. 1. - Corrélation quantitative entre la distribution relative des IRNA isoaccepteurs ef la fréquence des codons synonymes correspondants des mRNA connus chez E. coli.

Cette relation quantitative est également observée pour la partie moyenne de la glande séricigène (ou réservoir) qui produit la séricine et pour d'autres tissus du Ver à soie (Chevallier et Garel, 1979). C'est aussi le cas de l'appareil de traduction de l'hémoglobine dans le réticulocyte de lapin. En nous fondant d'une part sur les résultats de Hatfield ef al. (1979) pour les tRNA et le catalogue complet des codons des mRNA des alpha et bêta globines, nous avons trouvé un coefficient de corrélation linéaire hautement significatif pour 22 couples d'iso-tRNA et codons (Chavancy et al., 1979). 
En outre, la comparaison des séquences codantes actuellement connues - plus de 20000 codons - par l'analyse de correspondance, a permis à Grantham (1978) et Grantham ef Gautier (1980) de montrer que chaque génome est caractérisé par une fréquence particulière de codons synonymes : virus, mitochondries, bactéries, plantes ef animaux. Ainsi, les mRNA animaux présentent les traits communs d'une grande famille : fréquence élevée des codons terminés par $C$, quasi-absence des codons terminés par $A$ ef $C G$. L'analyse des 13 espèces de tRNA provenant de divers tissus animaux correspondant aux 20 codons des 4 acides aminés majeurs des protéines (alanine, glycine, leucine ef sérine) conduit à une corrélation élevée entre la distribution moyenne de ces iso-tRNA et celle des codons de l'échantillon des mRNA (Chavancy et al., 1979).

2. Fonction possible de l'adaptation des IRNA : optimiser l'efficacité de la traduction.

Quel que soit le processus de régulation de la biosynthèse des tRNA abordé ailleurs (Chevallier et Garel, 1979), nous nous interrogeons sur la nécessité à laquelle répond l'adaptation des tRNA. Vitesse et fidélité sont les deux paramètres fondamentaux de la dynamique et de l'énergétique du ribosome. Si un grand nombre de travaux ont mis l'accent sur l'interaction entre ces deux paramètres et les changements de population des IRNA tant in vivo que in vitro, aucun modèle de fonctionnement du ribosome n'a jusqu'ici pris en compte la distribution des IRNA acylés environnant le site $A$. C'est la raison pour laquelle nous avons été conduit à étudier un modèle de fonctionnement du ribosome dit «système d'essais et d'erreurs ».

Ce modèle (fig. 2) est fondé sur deux fonctions distinctes : réception ef comparaison. Le récepteur sélectionne au hasard une espèce acylée de tRNA - et une seule

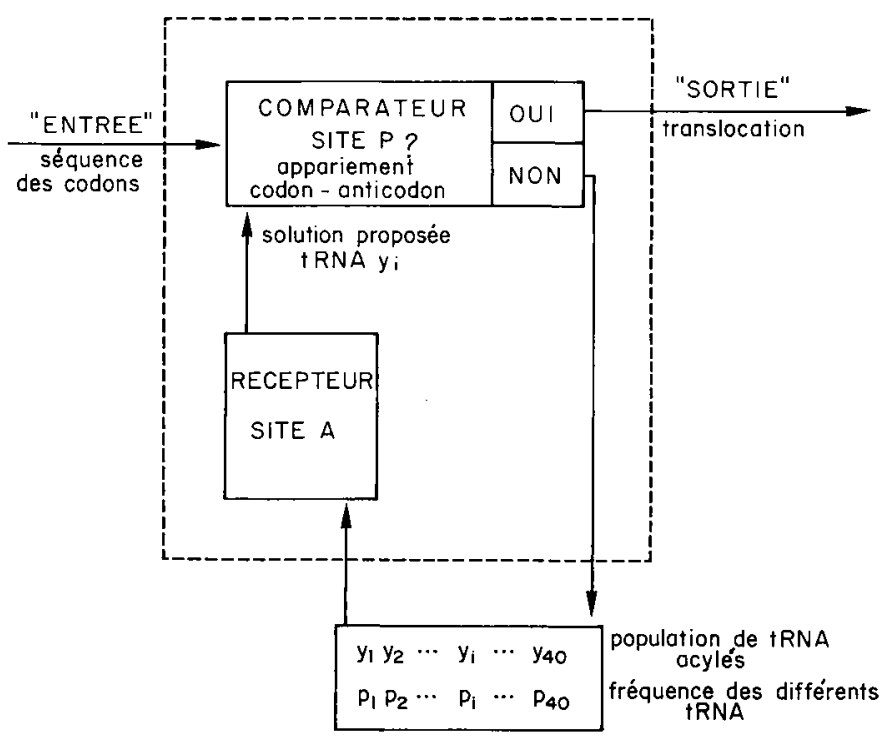

FIG. 2. - Modèle de fonctionnement stochastique du ribosome selon le système d'essais et d'erreurs.

à la fois - parmi la population qui entoure le ribosome à un moment donné. II la " propose » au comparateur qui procède à un essai ou comparaison avec le codon dis- 
ponible et fournit une réponse sur la pertinence de l'association codon-anticodon.

OUI, l'aminoacyl-tRNA essayé est correct. II est accepté ef la transpeptidation devient irréversible entrainant la translocation. Le ribosome est prêt pour un autre service faisant appel à un nouveau codon et à d'autres tRNA acylés.

NON, le tRNA proposé est incorrect et rejefé. Le récepteur devient accessible d̀ une nouvelle espèce acylée toujours choisie au hasard. Des essais successifs ont lieu jusqu'à l'acceptation finale.

Selon la conception classique, le site A du ribosome pourrait remplir à la fois la fonction de récepteur et celle de comparateur. Des données récentes (Peters et Yarus, 1979) permettent toutefois d'envisager un rôle discriminateur plus important au niveau du site $P$ que du site $A$. La fonction de comparaison pourrait alors être attribuée au site $P$ et dans ce cas, la transpeptidation serait réversible (Spirin, 1978 ; Peters et Yarus, 1979).

Le traitement mathématique de ce modèle stochastique (Pontier, 1970) montre que le nombre d'essais sera minimisé lorsque la concentration des tRNA est proportionnelle à la racine carrée de la fréquence des codons.

La corrélation parabolique de la figure 3 entre les concentrations absolves et non plus relatives des iso-tRNA du sécréteur et les codons du mRNA de la fibroine a été

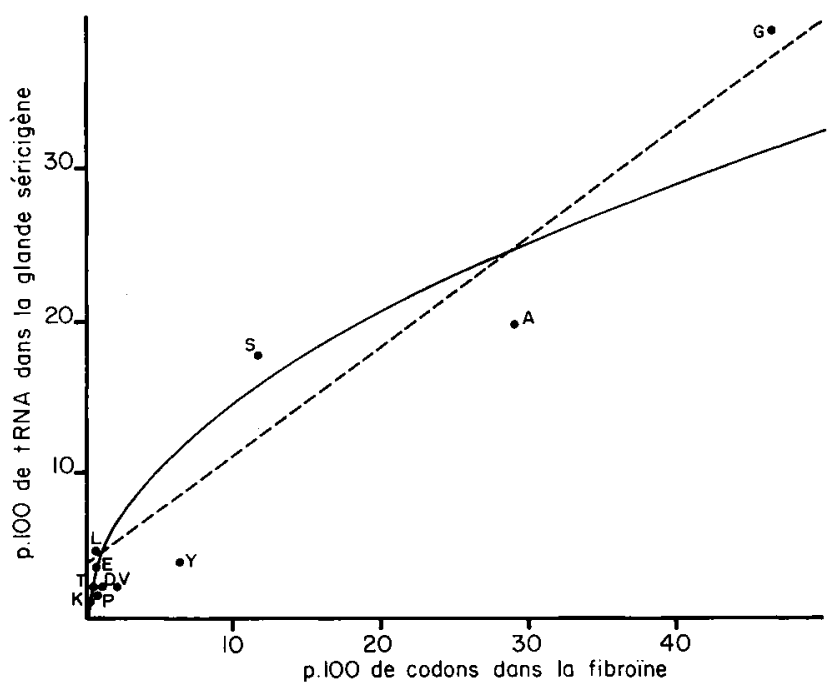

FIG. 3. - Corrélation de type parabolique entre la distribution absolue des iso-tRNA de la partie postérieure de la glande séricigène chez Bambyx mori (ou sécréteur) et la fréquence des codons du mRNA de la fibroine.

retrouvée pour le réticulocyte (Chavancy et Garel, 1981). Les données expérimentales disponibles semblent compatibles avec celles prévues par ce modèle. Ainsi, vitesse et fidélité du processus de l'allongement des chaînes protéiques seront optimisées par l'adaptation des tRNA.

Ce modèle de fonctionnement du ribosome permet de proposer une explication 
cohérente au fait que les populations de tRNA homologues sont toujours plus efficaces que des †RNA hétérologues sur la vitesse moyenne de traduction d'un mRNA. La figure 4 montre que la synthèse de fibroïne dans un système acellulaire de réticulocyte

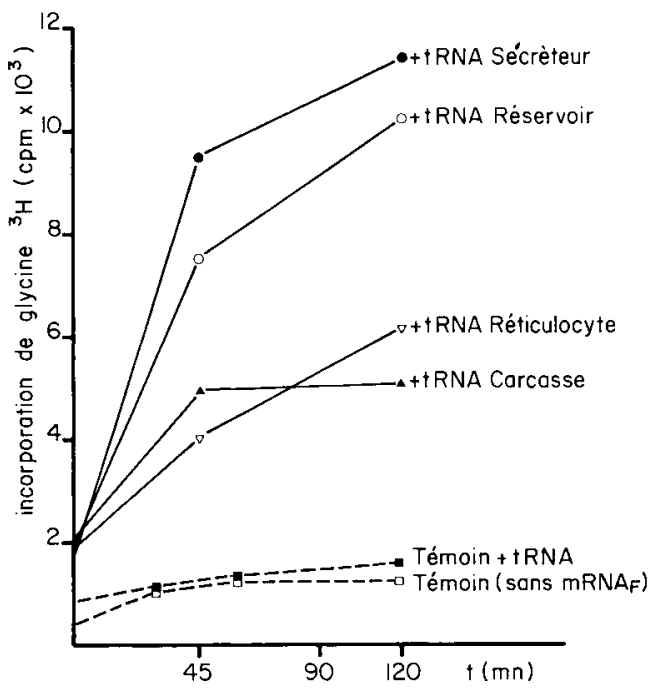

FIG. 4. - Cinétique d'incorporation de la glycine dans les protéines obtenues lors de la traduction du mRNA de la fibroïne dans un système acellulaire de réficulocyte de Lapin. Le système acellulaire est préparé et utilisé dans les conditions décrites par Pelham et Jackson (1976).
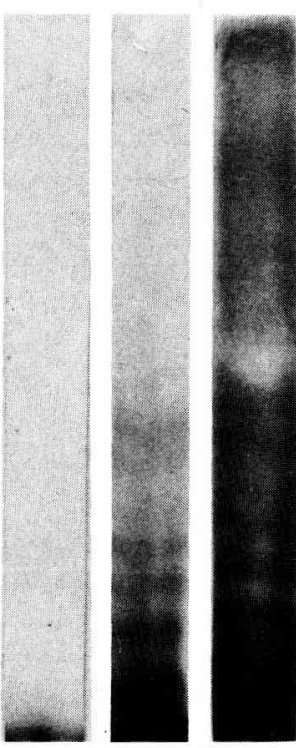

\section{fibroine}

FIG. 5. - Autoradiographie des protéines synthétisées dans un système acellulaire de réticulocyte de Lapin à partir du mRNA de la fibroine. Les protéines marquées par la $\left({ }^{3} \mathrm{H}\right)$-glycine lors de la traduction in vitro sont séparées par électrophorèse sur gel de polyacrylamide à 4 p. 100.1. - témoin (système acellulaire sans mRNA et tRNA exogènes) ; 2. - addition de mRNA de la fibroïne ( $1 \mu \mathrm{g}$ par essai) ; 3. - addition simultanée de mRNA de la fibroïne $(1 \mu \mathrm{g})$ et tRNA de sécréteur $(50 \mu \mathrm{g}$ par essai). Chaque essai est réalisé dans un volume final de $25 \mu$ l; l'incubation est effectuée à $37^{\circ} \mathrm{C}$ pendant $120 \mathrm{~min}$. 
de Lapin est maximale en présence de tRNA de sécréteur dans le milieu réactionnel. Dans ce cas d'ailleurs, la vitesse acquise permel l'obtention de molécules entières de fibroïne (PM 360 000) (fig. 5). Les vitesses moyennes $(0,7$ acide aminé/ribosome/s) sont toutes inférieures lorsque le système de protéosynthèse est supplémenté par d'autres sources de tRNA provenant du Ver à soie (réservoir et carcasse) ou du réticulocyte luimême.

En outre, notre modèle a une conséquence simple : les vitesses d'allongement individuel seront irrégulières et fonction de la distribution des codons. Les codons rares auxquels correspondent des tRNA mineurs ralentiront la vitesse de lecture. Or, la traduction in vitro du mRNA de la fibroïne conduit à l'accumulation de polypeptides plus courts que la fibroïne elle-même apparaissant sous forme de bandes discrètes sur électrophorèse (fig. 5). Ces polypeptides représentent des chaînes en cours d'allongement ef non des chaînes avortées (Chavancy efal., 1981 ; Lizardi et al., 1979). La traduction in vitro de la fibroïne est donc discontinue, elle marque des ralentissements ou pauses à des endroits précis du mRNA que nous interprétons comme les régions contenant une succession de codons rares qui encadrent les régions répétitives à codons fréquents.

\section{Conclusion.}

La population des IRNA dans toute cellule active, en croissance ou en développement, est ajustée à la demande en codons en voie de traduction. D'après le modèle proposé de fonctionnement stochastique du ribosome, l'ajustement des tRNA est nécessaire pour optimiser la vitesse moyenne en même temps que la fidélité. Cette conception d'un effet global d'une population de tRNA exclut l'hypothèse de la modulation assignant un rôle régulateur à quelques IRNA particuliers au niveau de l'allongement des chaînes protéiques.

6e Réunion du groupe Développement I.N.R.A., Clermont-Ferrand/Theix, 22-23 mai 1980.

\section{Références}

CHAVANCY G., CHEVALLIER A., FOURNIER A., GAREL J.-P., 1979. Adaptation of iso-tRNA concentration to mRNA codon frequency in the eucaryote cell. Biochimie, 61, 71-78.

CHAVANCY G., DAILLIE J., GAREL J.-P., 1971. Evolution des tRNA dans la glande séricigène de Bombyx mori au cours du dernier âge larvaire. Biochimie, 53, 1187-1194.

CHAVANCY G., GAREL J.-P., 1981. Does quantitative tRNA adaptation to codon content in mRNA optimize the ribosomal translation efficiency? Proposal for a translation model system. Biochimie (sous presse).

CHAVANCY G., MARBAIX G., HUEZ G., CLEUTER Y., 1981. Effect of tRNA pool balance on elongation rate and uniformity during translation of fibroin $m R N A$ in a reticulocyte cell-free system (submitted).

CHEVALLIER A., GAREL J.-P., 1979. Studies on tRNA adaptation, tRNA turnover, precursor tRNA and TRNA gene distribution in Bombyx mori by using two-dimensional polyacrylamide gel electrophoresis. Biochimie, 61, 245-262.

DAILLIE J., 1977. Synthèse des RNA de la glande séricigène du Ver à soie : adaptation à la production de fibroïne. Biol. cell., 29, 1-6.

GAREL J.-P., 1974. Functional adaptation of tRNA population. J. theor. Biol., 43, 211-225. 
GAREL J.-P., 1976. Quantitative adaptation of isoacceptor tRNAs to the codons of alanine, glycine and serine. Nature, 260, 805-806.

GAREL J.-P., GARBER R., SIDDIQUI M. A. Q., 1977. Polyacrylamide gel electrophoresis mapping of IRNA from the posterior silk giand and carcass of Bombyx mori. Biochemistry, 16, 36183624.

GAREL J.-P., VIRMAUX N., MANDEL P., 1970. Adaptation fonctionnelle des TRNA à la biosynthèse protéique : mise en évidence chez le cristallin. Bull. Soc. Chim. biol., 52, 987-1006.

GRANTHAM R., 1978. Viral, prokaryote and eukaryote genes contrasted by mRNA sequence indexes. FEBS Lefters, 95, 1-11.

GRANTHAM R., GAUTIER C., 1980. Genetic distances from mRNA sequences. Naturwissenschaften, 67, 93.

HATFIELD D., MATTHEWS C. R., RICE M., 1979. Aminoacyl-tRNA populations in mammalian cells. Chromatographic profiles and pattern of codon recognition. Biochim. biophys. Acta, 564, 414-423.

LIZARDI P. M., MAHDAVI V., SHIELDS D., CANDELAS G., 1979. Discontinuous translation of silk fibroin in a reticulocyte cell-free system and in infact silk gland cells. Proc. nat. Acad. Sci. USA, 76, 6211-6215.

PELHAM H. R. B., JACKSON R. J., 1976. An efficient mRNA-dependent translation system from reticulocyte lysates. Eur. J. Biochem., 67, 247-256.

PETERS M., YARUS M., 1979. Transfer RNA selection at the ribosomal A and P sites. J. mol. Biol., 134, 471-491.

PONTIER J., 1970. Traitement des informations chez les êtres vivants : systèmes à services par essais successifs. Bull. Math. Biophys., 32, 83-148.

PRUDHOMME J.-C., COUBLE P., 1979. The adaptation of the silk gland cell to the production of fibroin in Bombyx mori L. Biochimie, 61, 215-227.

SPIRIN A. S., 1978. Energetics of the ribosome. Prog. nucl. Ac. Res. mol. Biol, 21, 39-62.

SUZUKI Y., BROWN D. D., 1972. Isolation and identification of the mRNA for silk fibroin from Bombyx mori. J. mol. Biol., 63, 409-429. 